\title{
INTENCIÓN E INTENCIONALIDAD. APORTES PARA ACLARAR UN EQUÍVOCO
}

\author{
María Luisa PFEIFFER \\ $U B A$ - CONICET
}

Resumen: Mi propósito en este trabajo es precisamente detenerme en el modo de relación intencional, separar la intencionalidad, tal como es propuesta por la fenomenologia de la intención, con que suele confundirsela; precisar a qué apunta y mostrar cómo desde ella es posible incluso comprender dónde tiene origen la distancia marcada por las corrientes de pensamiento tradicionales. Para ello tomará como punto de partida la propuesta husserliana y los aportes de otros pensadores provenientes tanto de la filosofia como de la psiquiatria y procuraré hacer una sintesis que facilite materiales para volver a pensar este instrumento conceptual.

\section{Intencionalidad fenomenológica}

Sujeto y objeto siguen siendo tanto para la filosofía como para otras maneras "científicas" de acercarse a lo humano, dos conceptos oscuros y al mismo tiempo claves. Venimos constatando a lo largo de la historia del pensamiento el abismo entre uno y otro y la imposibilidad de franquearlo nos sume muchas veces en el escepticismo más feroz. La fenomenología, de la mano de Husserl, incorpora la intencionalidad a esta reflexión, como clave para poder pensar la relación sujeto-objeto desde una perspectiva alejada de un ingenuo realismo o un idealismo a rajatabla en que sólo "permanece" el sujeto ${ }^{1}$.

1 Es interesante ver cómo Mohanty que en ciertos aspectos difiere de lo que propongo en este trabajo, porque termina por acercarse a una interpretación "materialista» de Husserl, coincide en que la intencionalidad es la clave para "volver irrelevante" el planteo realismo-idealismo, cf. intervención en el debate en ZANER. R. "Passivity and Activity of Consciuousness in Husserl», Tyminiecka MT.(ed.), Analecta Husserliaina, Vol III, 218-219. 
«Realismo» e "idealismo» llevados a su máxima expresión sólo pueden priorizar uno de los extremos de la separación para quedarse con el lado sustancial que caracterice su modo de juzgar. La otra posibilidad que es la abrazada en nuestro tiempo, es el escepticismo.

Si bien me ocuparé de la intencionalidad es imposible hablar de ella sin tocar temas con los que está íntimamente unida como la intuición de las esencias, su relación con la psicología, la constitución del yo, la "realidad» del mundo; pero sólo me referiré a ellos de manera tangencial.

\section{El método husserliano}

Es necesario tener en cuenta la reducción trascendental husserliana, para comprender la intencionalidad, ya que por no hacerlo hay bastante confusión entre los fenomenólogos y los que no lo son cuando se refieren tanto a ésta como a la conciencia. Es más, como dice Bernet, los fenomenólogos no se mezclan casi en las discusiones actuales sobre esos $\operatorname{conceptos}^{2}$ y podemos agregrar que en razón de ello la confusión crece.

Lo que cuestiono es una intencionalidad reducida ella misma a acto "consciente", al acto de una conciencia que ipso facto adquiere estatus ontológico sustancial.

La reducción es solamente una parte del método fenomenológico, pero es aquella que permite comprender cómo actúa la conciencia y llegar a su "esencia" o para expresarlo más claramente caer en la cuenta de cómo "funciona" eso que llamamos concienca. En ella pasamos, según Husserl, de la radicalidad eidética a la radicalidad trascendentah, siendo ésta la que funda toda trascendencia en tanto y en cuanto lo que hay es dado en la conciencia en una dación originaria.

¿En qué consiste la reducción? Es el proceso por el cual se ponen entre paréntesis todos los datos, convicciones, interpretaciones, etc, que sustentan a los actos (cognoscitivos, volitivos, perceptivos, desiderativos, afectivos,

2 Recordemos que en esto coinciden los estudiosos de Husserl, la reducción junto con la epojé son los puntos claves de la epistemología fundada por Husserl, por consiguiente, su uso es conditio sine qua non para acceder a la postura fenomenológica. 
etc.), para volverse sobre los actos mismos y poder describir en qué consisten. El método husserliano propone realizar dos reducciones para alcanzar la "trascendentalidad" de la conciencia: la reducción psicológica o eidética y la reducción trascendental o fenomenológica. En la reducción psicológica o eidética se ponen entre paréntesis todas las experiencias o procesos particulares, se los "reduce» a "ideas». En la reducción trascendental o fenomenológica se ponen entre paréntesis las ideas (por idea entiendo: representación, «objeto", conceptos...) y se las "reduce" a "fenómenos" alcanzando de esta manera la conciencia su carácter trascendental; se accede así al "modus operandi» de la conciencia.

La actitud radical entonces pone entre paréntesis, en primer lugar al mundo "natural", la creencia en él y las proposiciones a que da lugar esta creencia, esto produce abstención de juzgar sobre la existencia espacio-temporal de los "hechos" o "datos". Aunque no podemos eliminar la actitud natural como vivencia, podemos no hacer uso de ella, hacer epojé y dejar así que el mundo pierda su carácter de existente, de dato inmediato, de supuesto. Entre lo que ha de ponerse entre paréntesis se incluye al yo y sus aportes. De esta manera el mundo y el yo pierden su carácter "ingenuo", "particular", «sabido" y pasan a ser conceptos, ideas, abstracciones, números, pasan a ser "experiencias". La denominada experiencia empírica no es "lo dado", es una interpretación sobre "lo dado", una especie de idea, de la que se hace epoje en el segundo paso metodológico.

Al practicar la segunda epojése pone entre paréntesis todo saber, toda referencia y conocimiento previo sobre «lo dado", todo lo que se pueda formular como juicio, todo lo que se pueda pensar, conceptualizar, calcular o formular. Lo que queda "entre las manos" luego de este segundo movimiento es el funcionamiento mismo de la conciencia, la "cosa misma» conciencia que era el objetivo del trabajo metodológico. Esta es la reducción trascendental donde se llega al enfrentamiento con la propia conciencia y su modus operandi. Y es ésta la que revela «lo dado" como dado, es decir como fenómeno; queda al descubierto que lo que se presenta, aquello a lo que ha de hacerse referencia está cruzado y rodeado por un horizonte "oscuramente consciente" resultante de una intencionalidad que lo atraviesa y envuelve significativamente. «Lo dado» se da como sentido y significación.

Esta segunda reducción posibilita enfrentarse a la dación fenoménica originaria, a lo constitutivo de lo dado, la conciencia alcanza la trascendencia 
como un modo de presentación del fenónemo. No hay contenidos de conciencia a los que se pueda hacer referencia luego de esta epojé, sino "fenómenos" y la descripción de los fenónomenos será la descripción de «lo dado».

La reducción invierte la dirección de la intencionalidad de la conciencia y suspende el mundo que ésta constituye ajena a todo método. A cada etapa de la reducción corresponde un plano intencional diferente, la reducción eidética deja ver una conciencia intencionante reflexiva, la reducción trascendental una conciencia intentionante pre-reflexiva. Esto nos permite descubrir que lo originario no es el cogito sino el fenómeno, lo originario no es la reflexión sino un mundo fenoménico poblado de sentido.

Cuando se confunde intencionalidad con intención, el método fenomenológico es utilizado sólo hasta el nivel de la reducción egológica o psicológica, es decir, todo queda reducido a la esfera de lo representativo, lo eidético, la esfera del yo como voluntad guiada por una intención o fin. Por consiguiente, el yo y sus facultades queda excluído de la epojé y es descrito como polo noemático o como auténtico sujeto.

Desde este planteo, hay algunos problemas tradicionales que siguen sin resolverse como el de la constitución del propio yo y del mundo, que resultan "construcciones subjetivas" a las que finalmente se termina "objetivando" para salvar la trascendencia. Estos problemas sólo pueden ser resueltos auténticamente desde un planteo de la intencionalidad que supere el nivel del sujeto o del yo. Esto significa que si bien Husserl plantea un métido en el cual es preciso pasar por la reducción eidética o psicológica, si nos quedamos en ella nos quedaremos en el ámbito de lo psíquico ${ }^{3}$.

\section{La intencionalidad}

La intencionalidad pre-reflexiva, "fungierende", aparece luego de la epojé del yo, cuando se ha practicado la reducción trascendental, cuando la conciencia se muestra en "carne y hueso". La intencionalidad es la "cosa misma" conciencia. Al llegar al nivel trascendental nos topamos con una conciencia

3 En su análisis del método fenomenológico J. San Martín muestra muy bien el alcance de estos dos tipos de reducción en las Meditaciones Cartesianas de Husserl, J., La estructura del método fenomenologico, UNED, Madrid, 1986 especialmente p. 33 
cuya principal característica es la de no ser una sustancia. Esto nos hace dejar atrás la idea de una conciencia asimilada de alguna manera al "alma", al "espíritu" o a la "razón". Descubrimos con Hussserl a la conciencia como "apertura a..." no como una "cosa" que tenga su dentro y fuera, es decir, que pudiera ser algo así como un recipiente contenedor de sus objetos. Nociones como las de inmanencia y trascendencia ya no pueden ser asimiladas a adentro y afuera, interior y exterior, sino que se comprenden a partir de un primer momento intencional que vuelve sobre sí mismo reconociéndose y en ese reconocimiento encuentra un límite, lo que está más allá de ese límite es lo trascendente, lo que está más acá es lo inmanente. Pero el límite no está dado, sino que como todo mundo de sentido, va variando todo el tiempo, va «cobrando forma» todo el tiempo. Esta conciencia «apertura a...» constituye «simultáneamente" conciencia y mundo y, sin ninguna mediación ni espacial ni temporal funciona como focalizadora, como razón. No hay entonces una intimidad privada donde se esconden los resultados de un acto constitutivo, no hay una distinción a la manera cartesiana entre la razón y sus objetos.

Es muy difícil expresar o describir este acto contitutivo intencional sin recurrir a imagenes y conceptos tradicionales, el resultado es muchas veces la aparición solapada del dualismo, como en la descripción de Montero Moliner: «las actividades conscientes del hombre están esencial y radicalmente volcadas en sus objetos, se funden con ellos de forma tal que sólo mediante una penosa abstracción podemos distinguirlos" ". Montero Moliner sigue hablando de "volcar" y "fundir" aunque reconoce que reducir la conciencia a objeto supone una "penosa abstracción». Esta abstracción es la que posibilita separar la conciencia como noesis intencionante de su noema intencionado. Es preciso dejar en claro aunque resulte redundante, que esa conciencia intencionante que aparece en la última epojé no es una sustancia sino intencionalidad, es decir tensión, apertura a..., pura actividad constitutiva. "Lo primero, dice Merleau-Ponty, no es la conciencia y su Ablaufsphänomen con sus hilos intencionales distintos, sino el torbellino que ese Ablaufphänomen esquematiza, el torbellino espacializante, temporalizante" ${ }^{5}$.

¿Cómo estar parado en un torbellino? ¿Cómo «atrapar» algo en un torbellino? ¿Cómo describir esta actividad de la conciencia? Cuando Merleau-Ponty se repon-

4 Montero Moliner, Fernando, Retorno a la fenomenologia, Anthropos, Barcelona, 1987. p. 324. (subrayado mío)

5 Merleau-PonTy, M., Le visible et l'invisible, Gallimard, Paris, 1964, p.298. 


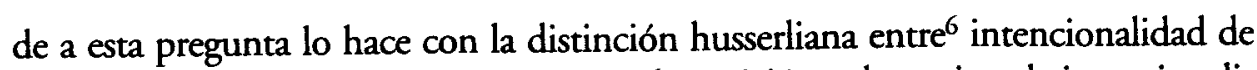
acto que es la de nuestros juicios y tomas de posición voluntaria, y la intencionalidad actuante o fungierende. "la que hace la unidad natural y antepredicativa del mundo, que aparece en nuestros deseos, valoraciones, paisajen ${ }^{7}$. Distingue entonces en el acto intencional de la conciencia lo que se llama intención es decir los actos psíquicos dirigidos hacia un objeto dado, que requieren algún nivel de reflexión objecivante, y la intencionalidad propiamente dicha que muchos denominan pre-intencionalidad para evitar esta confusión con la intención ${ }^{8}$. La intencionalidad de acto, la que emite juicios, valora voluntariamente, es un tender a un fin que ya está allí, un fin que va siendo objetivado por ella como fin querido, o conocido, o sentido, es el Ablaufphänomen de que hablaba más arriba que ya ha sido sometido a la reflexión en mayor o menor medida; la fungierende, es pre-reflexiva, corporal, fundante y posibilitante de la otra, al intencionar lo hace originariamente, conformando sentido, de manera operante y nunca de manera terminada, es carne ${ }^{9}$ espacializante y temporalizante. El resultado de la intencionalidad prereflexiva son mundos intersubjetivos, el de la de acto la imagen de esos mundos, su concepto, su nombre, su representación, un mundo recortado sobre el primero, un mundo subjetivo-objetivo. Podríamos diferenciar ambos mundos llamando a uno mundo (conjunto de mundos) intencional y al segundo mundo intencionado, el primer mundo es abierto, no acabado, el segundo tiene límites, el límite de su propia objetividad, de la intención conformadora con tinte «subjetivo» (del yo).

En el actuar de la intencionalidad operante no hay «intención", "finalidad" conformadora de algo que ya está allí sino la fundación del allí, del algo y del propio acto intencional, no es reflejo de una verdad previa, sino la realización de una verdad. La primera intencionalidad se expresa por la palabra, la segunda por el discurso, el juicio, que "habla" sobre la palabra. La palabra no dice si algo es o no es, simplemente expresa, más allá o más acá de la reflexión, lo que "sabemos" antes de "conocerlo", por eso muchas veces dice más de lo que el

6 cf. Merleau-Ponty, M., Phénoménologie de la perception, Gallimard, Paris, 1945, p. 490.

7 Merleau-Ponty, M., 1945, p.XIII.

8 Para Walton se dan dos posturas en el sujeto, la reflexiva que es transparente-trascendental y la corporal que es natural-opaca. Cf. WALTON, R., Husserl, mundo, conciencia y temporalidad, Almagesto, Bs. As., 1993

9 El vocablo carne es utilizado por Merleau-Ponty, uno de los mas fieles seguidores de Husserl, para referirse a esa conciencia corporal que intenciona antes de toda injerencia volitiva y racional 
que habla podría definir, «dice» más o menos de lo que la intención del hablante se propone. La palabra es fundante, originante, develadora, el discurso es analítico, valorizador, verificador. La palabra, el «verbo», es el lenguaje del artista, del escritor que siempre «dice más de lo que dice», del poeta que dice lo indecible, del filósofo que habla de lo que no se puede nombrar.

La actividad intencional prereflexiva no es de un sujeto sino que es intersubjetiva, esto hace que los mundos constituidos sean muchos y de todos y no un mundo propio. Sin embargo esto que se dice tan fácil es lo que acarrea mayores dificultades para ser pensado. La cuestión del otro y de lo otro que se manifiesta en la constitución intersubjetiva plantea dificultades que se ven muchas veces con más claridad cuando esa constitución falla, como es el caso de los modos patológicos de habitar el mundo. En un intento de descripción podemos decir que la intencionalidad pre-reflexiva es una co-actividad en la que el mundo (los mundos) va adquiriendo sentido, un sentido compartido, que no proviene de nadie en particular y proviene de todos en general. Es lo que Husserl llama "mundo de la vida".

Al recurrir a la locura en tanto patología, vemos que no afecta al nivel reflexivo de la conciencia, sino a la intencionalidad pre-reflexiva, no afecta a las intenciones sino a la intencionalidad, y eso es lo que diferencia a la locura de la filosofia. La locura cuestiona el nivel de lo pre-reflexivo, la filosofia el de la reflexión. Desde el planteo socrático, la duda es para el filósofo un método que le permite disponer el ánimo para el acceso a la verdad, en cierta manera incluso el método fenomenológico es un poner en duda análogo al socrático. Lo primero que exigía Sócrates era dudar hasta de sí mismo ${ }^{10}$. Sin embargo, el mismo Sócrates advierte que este es un camino lógico, ningún filósofo duda como filósofo cada mañana que la calle estará allí cuando salga de su casa; su duda es metódica, reflexiva; el loco por el contrario duda de que la escalera "esté puesta», su duda alcanza el nivel originario que es el nivel de la creencia, nivel que Merleau-Ponty llama "fe perceptiva" y Blankenburg "evidencia natural» y en el campo de la sociología Guiddens "seguiridad ontológica" ". La duda filosófica, "metódica» como la llamó Descartes, exige esta actitud creyente, confiada, de que el mundo en que estoy es un mundo "común" y eso basta. Eng compara la actitud del loco con la «admiración" filosófica y propone una terapia para con-

10 Esta actitud es muy cercana a la epoje del yo husserliana.

11 Guiddens como otros autores provenientes especialmente de la sociología han considerado que la modernidad ha puesto en cuestión esta actitud reemplazándola por la de fiabilidad. 
vertir la duda vital en duda reflexiva, lo que significa recuperar el nivel intencional pre-reflexivo ya que el loco lo ha perdido y sólo se mueve al nivel de las intenciones reflexivas ${ }^{12}$. El mundo del loco tiene disociada su intencionalidad, sólo pone en práctica la reflexiva, por eso el suyo se convierte en un reflexionar sobre nada, sobre vacío. Una actitud habitual en el esquizofrénico por ejemplo, es "copiar" los gestos de los otros en determinadas circunstancias: cuando conversa con un amigo, cuando va a bailar con su novia, etc. Las conductas son resultado de actos intencionales y no de actos intencionantes.

El nivel originario puede ser objetivable en tanto y en cuanto los acto de la intencionalidad operante pueden ser convertido en concepto. Ella está siempre en actividad constitutiva que sólo podemos atisbar, descubrir como aquello que siempre se nos escapa, pues aunque podemos detener al torbellino para describirlo o analizarlo sigue "funcionando" ${ }^{13}$. $\mathrm{Al}$ uobjetivar" se ve "funcionar» a la conciencia como algo ajeno a lo que estamos haciendo, se re-flexiona, se vuelve sobre lo que la conciencia está haciendo y se acompaña ese momento intencional originario dándole nombre, categoría, valor. Sin embargo, esto no siginifica que este volverse sobre la actividad intencional de la conciencia esclarezca definitivamente en qué consiste esa actividad, esta objetivación, como cualquier otra, sólo nos revela la opacidad, la inagotabilidad de eso que va adquiriendo carácter de objeto. También nos revela su trascendencia pero, tomando la expresión de Barbarras «lejos que la trascendencia sea la propiedad del objeto, el objeto es una dimensión de la trascendencia, es decir uno de sus modos de manifestación o de fenomenización» ${ }^{14}$.

\section{La psiquiatría}

Es interesante ver como la confusión intencionalidad-intención aparece notablemente expuesta al usar la metodología fenomenológica para elucidar problemas como los presentados por la psiquiatría. Giudicelli, uno de los más

12 ENG, ERWIN, «Los soldados en la juventud, los veteranos en la vejez. El cuerpo como conciencia” en RovaleTtI, M.L., (ed), Fenomenologia y corporalidad, Ciclo Básico Común UBA, Buenos Aires, 1996.

13 Fungierende significa funcionante, que está funcionando.

14 BaRBARAS, RENAUD, «De la parole à l'être: le problème de l'expression comme voie d'accès à l'ontologie" en AAVV, MERLEAU-PONTY, La philosophe et son langage, Recherche sur la philosophie et le langege, Grenoble, 1993, p 77. 
reconocidos psiquiatras franceses que han adoptado para su práctioca criterios provenientes de análisis fenoomenológicos, nos proporciona una descripción de su ruta epistemológica que vamos a examinar detenidamente para mostrar cómo queda preso de la reducción eidética y cómo reduce la intencionalidad a la intención de un yo guiado por una finalidad «subjetiva».

Giudicelli dice: “Me despojo entonces de las intencionalidades vacías del discurso corriente, ....intento atrapar el ser mismo del hecho psicótico tal cual lo intuyo, efectuando un acto intencional y subvirtiendo lo empírico en lo cual estoy inmerso en mi práctica diaria». Con esta primera frase "Me despojo entonces de las intencionalidades vacías del discurso corriente», quiere indicar que realizará una epojé, dejando de lado todo aquello que hasta el momento le permitió comprender o no comprender la enfermedad ${ }^{15}$; acusa a los discursos corrientes de carecer de intencionalidad, en realidad todo discurso proviene de una intencionalidad en tanto y cuento proviene de un mundo conformado de alguna manera, de lo que puede carecer es de intenciones con algún valor, que es a lo que en última instancia se refiere Giudicelli "intento atrapar el ser mismo del hecho psicótico tal cual lo intuyo" esta frase es clave ya que está pensada desde la concepción del "hecho" como sustento de un "ser mismo". Giudicelli da cuenta de su intento de apoderarse de algo "exterior" (el ser mismo) a la conciencia que ha sido atrapado por ella intuitivamente, es decir "directamente", sin intermediario. Esto último sería la manera de escapar del hiato. La conciencia y aquello que intenta atrapar (el ser mismo del hecho) ya están "fundidos» en la intuición. Conciencia y hecho son dos sustancias separadas que se funden (recordemos que Montero Moliner usó el mismo verbo aunque él lo aplicaba al objeto) Queda claro cómo vuelve a aparecer aqui el hiato de que hablaba más arriba y la dificultad de saltarlo incluso con conceptos tan discutidos como el de intuición. Y agrega: "efectuando un acto intencional y subvirtiendo lo empírico en lo cual estoy inmerso en mi práctica diaria", ¿qué significa esto? que ese "algo o alguien" esa "conciencia", que atrapó el hecho psicótico por la «intuición» efectúa un "acto intencional», que aquí significa «un acto de reflexión" con un cierto sentido o interés, sobre aquello que intuyó. Lo empírico recupera su carácter de hecho bruto, sin significación que debe ser puesto en contexto por el acto intencional. Este corto texto nos muestra cómo utili-

15 No tendremos en cuenta aqui el hecho de que ninguna intencionalidad puede ser vacía, tomaremos la expresión como poco feliz, dado que a fin del mismo capítulo afirma "nunca se da una "nada" fenomenológica en la intencionalidad de la conciencia". 
zando la terminología fenomenológica el planteo vuelve a ser el de una conciencia separada de algo «exterior» a ella a lo que se dirige para darle determinada significación.

Sigo utilizando este ejemplo para mostrar la confusión que suele establecerse entre las dos operaciones de la conciencia, olvidando la primera en aras de la segunda ${ }^{16}$. Lo que Giudicelli llama aquí acto intencional es en realidad un acto de intención, incluso más explícitamente: es el ejercicio de una intención metódica lo que lleva a cabo. La intencionalidad queda reducida a un reconocimiento del cogito, al acto de una conciencia subjetiva identificable con un yo que se vuelca sobre «algo» aprehendido previamente por la «intuición».

Giudicelli, que hace excelentes descripciones de actos intencionales constitutivos de la temporalidad, en sus pacientes, no logra deshacerse del enfoque egológico al "traducirlos" en conocimientos "objetivos»: En su análisis sigue al nivel de la primera reducción.. La consecuencia de no realizar la segunda epojé es seguir preso en la dualidad cognoscitiva, esto aparece muy claramente un poco más adelante cuando dice "lo inteligible y lo sensible están ligados en las evidencias correlativas al mismo tiempo que son definidos por la diferencia de los objetos a que apuntan. Sobre la base de una intuición sensible, el conocimiento se realiza por la idealidad a que apunta» ${ }^{17}$.

Esta descripción del acto intencional podemos encontrarla en muchos de los psiquiatras que adoptan la fenomenología husserliana, y está muy cerca de la que hacen autores como Searle por ejemplo o el mismo Brentano. Acentúan la descripción que es hecha por un sujeto, por ello pretenden poner entre paréntesis el síntoma junto con todos los supuestos científicos, pero éstos se les cuelan por la ventana junto con la objetividad, ineludible si la descripción es hecha desde un sujeto. Por ejemplo no pueden dejar de lado la causalidad, -la noción de causa se introduce oculta bajo principios formales-, ni en última instancia la de realidad.

16 Reitero que el primera y segunda no tiene que ver con su sucederse en el tiempo sino con su situación en la constitución del mundo luego del análisis efectuado por el método fenomenológico. Hay una constitución del mundo pre-reflexiva que «sostiene» a la reflexiva, por eso llamo primera a la una y segunda a la otra.

17 Giudicelli, S., NAUdin, J., DASSA, S., "La implicación husserliana en psicopatología", en RovaletTI, M.L., (ed.), Psicologia y psiquiatria fenomenologica, Facultad de Psicología, UBA, 1994, p. 265. 


\section{Brentano y Searle}

La relación entre la concepción de la intencionalidad en estos dos autores y su comparación con la que comienza a apuntar en Husserl y es desarrollada por los fenomenólogos posteriores, pone de manifiesto la confusión que pretendo iluminar. Sabemos que Brentano toma prestado a la escolástica tardía, el concepto de intencionalidad para diferenciar los actos psiquicos de los físicos. Para los escolásticos había dos maneras de "existir» la cosa, una real y otra intencional. La intencionalidad designaba «la presencia de un objeto en la conciencia", que era un doble del objeto real. Brentano traduce estos modos de "existir" como intramental y extramental. Por ello puede decir que la "realidad" o la "extramentalidad" no es una condición necesaria para que algo sea considerado como un objeto intencional ${ }^{18}$. Todo objeto intra o extramental "esta» intencionalmente en la conciencia como conocido, querido, representado, percibido, etc., y por consiguiente debe ser distinguido de ella.

Searle coincide con este análisis en la concepción de una conciencia sustancial y de un mundo "fuera» o "dentro» de ella. La reducción es epojé del mundo externo, y su análisis de la intencionalidad se limita al del mundo interno de la vida mental del sujeto ${ }^{19}$.

Como lo hizo Brentano en su momento, concibe la intencionalidad de una manera psicológica, como una particularidad de la naturaleza interna de ciertos estados mentales. Tanto para Brentano como para los analíticos la mente es semejante a la conciencia cartesiana, algo interior que contiene alguna cosa ${ }^{20}$. En tanto y en cuanto la fenomenología husserliana participa de las estructuras del pensamiento cartesiano y subraya lo más posible sus tendencias solipsistas, permite a un autor como Searle explotar los análisis fenomenológicos de la

18 Esto es lo que permite que se traduzca indistintamente cosa o realidad

19 También Searle diferencia intención de intencionalidad. Para él esta distinción tiene que ver con la acción, la intención sería la inclinación a hacer algo, mientra la que intencionalidad sería un estado que tiene que ver con la intención en acción. La intencióon es conformada con un acto que pretende algo, que tiene un fin, La intencionalidad es la direccionalidad de los actos mentales, no implica ninguna acción.

20 En general encontramos en la corriente analítica un esfuerzo por mantener su reflexión en la esfera de "lo mental", del conocimiento, y no mezclar sus problemas con "cuestiones metafísicas", como podría ser la cuestión ontológica acerca de "la realidad". Sin embargo estas cuestiones se deslizan cuando empleamos adjetivos o adverbios como externo-interno, exterior-interior. 
intencionalidad y alimentarse de ellos ${ }^{21}$. Al hacer una lectura cartesiana de Husserl, insistiendo sobre la diferencia radical de las esferas inmanente-intencional y trascendente-natural, es posible mantener que la fenomenología de Husserl se apoya sobre una estructura dual clásica. Podemos decir entonces que si la filosofía analítica discute con Husserl y muchas veces coincide con él, es precisamente acentuando lo que se ha percibido en los trabajos husserlianos como un defecto o una manera de no llevar hasta el fin la mayor exigencia de su pensamiento, esa que fuera expresada como «llevar a la experiencia, muda todavía, a la expresión pura de su propio sentido" ${ }^{22}$.

El elemento fundamental para el análisis de la intencionalidad es para la corriente analítica el noema husserliano. Éste es comprendido y utilizado como un concepto y puede jugar tres papeles:

- el de una forma abstracta a la que sólo podemos acceder por la lógica;

- el de una estructura formal desde la que el noema se dirige a los objetos;

- el de un sistema complejo de determinaciones de las que el objeto sería una función. Cualquiera de estos tres roles, forma, estructura, sistema, parecen atribuirle las características de un operador lógico independiente, hasta el punto de opacar las funciones de la conciencia. La estructura dual de relación hombre-mundo, se convierte así en ternaria: concienciaobjeto-sentido. Este planteo es compartido por Searle ya que afirma que para conocer lo que es un estado intencional es suficiente conocer tres factores: 1.cuáles son las condiciones de satisfacción (el objeto intencionado), 2.en qué aspecto el contenido representativo representa a estas condiciones (el sentido o la materia del acto intencional) y 3 . cuál es el modo del estado intencional en cuestión (nivel de conciencia).

Si tomamos la descripción del acto intencional que realiza Husserl en sus Investigaciones Lógicas encontramos un fuerte paralelismo con la realizada por

21 Los análisis que pretenden este tipo de acercamiento, como el de A.Z. Bar-On, "Who, Searle or Husserl?", o DreYFus, H., Husserl, Intentionality and Cognitive Science, se apoyan especialmente en las descripciones de la intencionalidad que hace Huserl en sus primeras obras, especialmente Investigaciones Lógicas.

22 Hussert, E., Méditations Cartesiennes, Vrin, Paris, 1953, p.33, Merleau-Ponty la retoma una y otra vez como "leiv motiv» de su reflexión. 
Searle. Sin embargo el punto de partida de Husserl es diferente del de Searle. Si hay algo en lo que insiste Husserl es que el método fenomenológico, por medio de la epojé no neutraliza el mundo sino la representación del mundo. Mientras Descartes haciendo un planteo diferente parece deslizarse imperceptiblemente a la esfera del $\operatorname{ser}^{23}$, Husserl pretende quedarse en la del conocer: «Lo que Descartes pone seriamente en duda, nosotros lo ponemos en cuestión".

De modo que es correcto afirmar que sólo puedo eliminar los caracteres noemáticos de la re-presentación del mundo, pero el mundo sigue a la par de este acto metódico constituyéndose en tal, no porque el mundo sea algo ajeno al sujeto que pregunta por él, sino porque su "realidad" es intersubjetiva y prereflexiva. Si el movimiento intencional se "detiene" en un cuadro, como se hace con una película y se lo analiza por una exigencia metódica, se descubren en él dos polos, la noesis y el noema pero también que además de haber sido constituido por ese movimiento dador de sentido que es la intencionalidad, se puede tener una imagen de él, re-presentarlo, re-cordarlo, pro-yectarlo, darle un tono afectivo: quererlo, odiarlo, desearlo, rechazarlo. Pero, mientras se realiza ese "trabajo" de análisis, se sigue intencionando junto con los otros, completando, modificando, removiendo, reafirmando el sentido que se está objetivando. Más allá de toda capacidad reflexiva, hay mundos que escapan para siempre a cualquier análisis o representación..

Cuando Searle describe la intencionalidad, recurre al análisis de los actos del habla con que se expresa lo mental. Crea así, paradójicamente, un nuevo cartesianismo en que ni siquiera están ausentes las connotaciones sustancialistas del originario. La mente, en efecto, es el cerebro humano con todas sus reacciones nerviosas al mejor estilo biologista. Para Searle el mundo de la experiencia inmediata es la representación. Pero al mismo tiempo esa representación implica un mundo fuera de ella, ¿y de dónde proviene esta implicación? para Searle de una "idea», de un concepto. Si tomamos en cuenta el movimiento intencional que se está apareciendo como experiencia inmediata, la implicancia sólo es la manifestación de una creencia prereflexiva, pre-representativa. Si es verdad como dice Searle que descriptivamente percepción, creencia, etc., son tomadas perpetuamente en un conjunto comportamental y existencial global, cognitivo, volitivo, afectivo... y que un estado intencional dado «no determina sus condiciones de satisfacción

23 Cf. MARfa LUISA PFEIFFER, "El límite de la duda», Revista Latinoamericana de Filosofia, Buenos Aires, Vol IV, Num. 1, 1978. Esto es corroborado por la posición ética de Descartes. 
mas que en relación con todo otro tipo de estado intencional ${ }^{24}$, parece absurdo hacer reposar esa red sobre un fondo que él mismo caracteriza como desenlazado de intencionalidad aunque "explícitamente mental" ${ }^{25}$. Leyendo su capítulo titulado Background (subsuelo) tenemos remembranzas de una descripción de la intencionalidad operante, pero ese Background para Searle es el mundo «real».

El dualismo es así recuperado, aunque este ya no se da entre una sustancia material y una espiritual, entre cosas extensas y corpóreas y cosas inmateriales e inextensas, sino entre un cuerpo extenso que obedece y un cuerpo extenso que manda, un cuerpo extenso que recibe intenciones y una mente que las produce como representaciones. Para Searle la distinción husserliana entre la materia y la cualidad de un acto sería equivalente a la diferencia introducida por Austin entre el contenido proposicional y la fuerza ilocutoria. El fundamento de la intencionalidad es la representación y el hecho que un estado intencional esté dirigido sobre un objeto significa que éste encierra la representación de sus condiciones de satisfacción. La intencionalidad consiste en una "dirección de ajuste» entre el espíritu y el mundo de tal manera que un estado intencional funciona de manera similar a un acto del habla.

"Representación" y "causalidad» son la clave de toda esta comparación. Son pivotes sobre los que gira el análisis de Searle que podríamos asentar sobre:

1. Una noción clásica de verdad como adecuación del espíritu y la cosa. Esta adecuación aparece en Searle como ajuste entre la interioridad de lo mental y la exterioridad del mundo: eso que es representado por el estado intencional, viene a satisfacerlo.

2. Hay una relación causal entre un estado mental interno y el mundo exterior. No por nada Searle presenta su teoría como «una versión de realismo naif" 26 .

El planteo neo cartesiano de la filosofía analítica compartido por Searle, está cuestionado desde la intencionalidad "fungierende" ya que es un ámbito totalmente independiente de la predicación; la vivencia primordial resiste al

24 SEARLE, JOHN R., L'intentionalité. Essai de philosophie des états mentaux, Les Editions de Minuit, Paris, 1983, p. 172. (cito la versión francesa de Intentionality. An essay in the philosophy of mind, Cambridge University Press, Cambridge, 1983)

25 SEARLE 1983 (versión francesa) p. 189.

26 SEARLE 1983 (versión francesa) p. 78. 
concepto, pensado exclusivamente como representación. No ignora el enunciado ni la objetivación reflexiva pero su expresión más propia es simbólica, aproximada y provisoria.

Para poder acceder a este modo de expresión, y realmente dejar atrás el planteo dualista es preciso tomar en cuenta la dimensión corporal de la intencionalidad, caer en la cuenta que "es carne y no noema frente a un noema" ${ }^{27}$. Si olvidamos la ambigüedad a que nos lleva la condición corporal de todos nuestos actos, caemos en la trampa searliana que buscando escapar del planteo dualista convierte al humano en un organismo y a todas sus reacciones en reacciones orgánicas ${ }^{28}$. En este esquema la intencionalidad funciona como causa, es la capacidad del cerebro de guiar al "cuerpo» (organismo biológico) ${ }^{29}$ a colmar sus carencias. La carencia que manifiesta la intencionalidad es colmada por el mundo "exterior", el mundo "real».

\section{Conclusión}

Si la intencionalidad que con Merleau-Ponty llamamos operante es conciencia corporal es posible detenerla, recortarla para el análisis, es decior hacer epojé. La epojé como movimiento metodológico es válido, no lo es como modo de vida, como modo de ser en que refiero una y otra vez al noema por el ejercicio de la noesis, olvidando que toda referencia es constante y necesariamente interferida por los otros. El mundo es intersubjetivo, la intencionalidad es intersubjetiva, incluso la ciencia debería reconocer su carácter de intersubjetiva ${ }^{30}$.

27 Merleau-Ponty, M., 1964, p. 298.

28 En efecto, lo que Searle establece como respuesta es meramente mostrar que hay una correlación empírica entre los fenómenos intencionales y los estados del cerebro, pero esto ya lo sabían Descartes y Leibnitz. Por otra parte también para Brentano los procesos pasíquicos dependen de procesos orgánicos, hay que analizar las condiciones materiales y lo vivido. Propone asi dos psicologías: una genéntica o fisiológica y otra psicognósica: psicología descripriva o fenomenología descritptiva.

29 Cf. los comentarios de Mètraux respecto del materialismo científico, él considera que Searle enraiza la intencionalidad en dicho materialismo, y que en ese momento deja los estados, sucesos, procesos y fenómenos psíquicos reducidos a estados, sucesos, procesos y fenómenos físicos por un simple pase espistemológico. Por ejemplo en "L'intentionnalité et le problème de la réduction de la psychologie», Etudes phénoménologiques, Ousia, N. ${ }^{\circ} 4,1986$, pp. 75-98

30 El concepto de ciencia de Feyerabend va en ese sentido. Cf. FEYERABND, PaUL, Adiós a la razón, Tecnos, Madrid, 1996. 
Cuando acudimos a la psiquiatría encontramos allí ejemplos iluminadores acerca del rol de la conciencia en la vida de los hombres, puesto que los disturbios patológicos no son ajenos a las formas comunes de la existencia humana. Eng que nos propone como terapia transformar al loco en filósofo, se apoya en esta aproximación a la conciencia. Lo que lo hace posible es que para el loco el mundo se constituye en la soledad de la conciencia racional. ¿acaso hay algo más parecido a esa descripción de la locura que la filosofía cartesiana? Pero, lo de Descartes era una filosofía, no un estar-en-el-mundo. El único modo que tuvo Descartes de recuperar el mundo perdido fue recurriendo a otro, es decir estuvo obligado a volver a una situación originaria de co-presencia. El filósofo debe hacer, por consejo de Descartes, lo que el enfermo, retirar los hilos intencionales que entretejen los sentidos de los mundos que compartimos. El mundo que queda entre sus manos es un mundo detenido en el momento de la objetivación, la suya es una situación de extrañeza y alienación, una situación que crea un equilibrio ficticio o más bien ficcional. La pregunta es, si este es el único método filosófico posible, si no se puede escapar de la crítica. Pero la dificultad es otra, es recuperar para la filosofía el momento previo a la reflexión, es encontrar el modo de evitar esconderse tras el confort de una palabra ya dicha o de un acto prefabricado, cómo arriesgarse a «la ambigüedad en sus infinitos tonos ocultos, como en un texto onírico, metafórico, impredecible y polidimensional" ${ }^{31}$. La filosofia y la locura han caminado muchas veces de la mano de aquellos que han pretendido reducir el mundo a una única representación por más globalizada que fuera, no de la de aquellos que han hecho del mundo mundos plurales.

31 Callieri, Bruno, «El símbolo y su riesgo psicótico», en Rovaletti, ML, (ed), Psicologia y psiquiatria fenomenológica, Facultad de Psicologia, UBA, 1994, p.257. 